\title{
Bending of CNTs Under The Partial Uniform Load
}

\author{
Mustafa Arda ${ }^{* 1}$ and Metin Aydogdu ${ }^{1}$ \\ ${ }^{I}$ Trakya University, Mechanical Engineering Department, Edirne, 22030 TURKEY \\ *Corresponding Author: mustafaarda@trakya.edu.tr
}

Received date: July 2016

Accepted date: August 2016

\begin{abstract}
In the present work, bending of CNT has been carried out. Effects of the nonlocality, characteristic length ratio and magnitude of the external uniform load have been investigated. Nonlocal Euler-Bernoulli Beam model has been used in the formulation. Present results could be useful for design of nano-sensor applications.
\end{abstract}

Keywords: Static Analysis, Radial Deflection, Nonlocal Theory, Partial Load.

\section{Introduction}

Carbon Nanotubes (CNTs) have many potential usage areas like nano-electromechanical systems, nano-sensors, nano-manipulators, etc. Especially static deflection of CNTs under the effect of partial uniform load can be used in nano-sensor technologies [1]. Radial displacement of CNT can be changed with the temperature or magnetic field changes. Continuum and discrete model have been used in modeling of CNTs. Classical continuum mechanics couldn't have been successful in nano-scale applications because of length scale. Nonlocal Elasticity, was established by Eringen [2-4], is a continuum model which includes the size effect with assumption that is "stress at a point in the continuum is the functional of stress field in every point of the continuum". By this way, a continuum model which incorporated both continuum and discrete model results had been established.

Static analysis of CNTs under the effect of external load has been investigated by scientists over the years. Dequenes et al. [5] studied carbon nanotube based nanoelectromechanical switches by using molecular dynamics(MD), linear and nonlinear continuum beam theories and combined molecular dynamics and continuum theories. Wang and Shindo [6] firstly developed nonlocal continuum theory for static and buckling analysis of CNTs. Gong et al. [7] investigated static and dynamic characteristic of CNT using molecular dynamics. Reddy and Pang [8] reformulated the equation of motion of the Euler-Bernoulli and Timoshenko beam theories using the nonlocal differential constitutive relations of Eringen and studied the static bending, vibration and buckling responses of beams at different boundary conditions. Aydogdu [9] developed a generalized nonlocal beam theory for bending, buckling and free vibration of nanobeams. Civalek et al. [10] presented the static bending analysis of CNTs using nonlocal Euler-Bernoulli beam theory and differential quadrature method. Torsional static analysis of CNT in an elastic medium under the different torque loads investigated by Arda and Aydogdu [11]. Static and buckling analysis of cantilever CNTs carried out by Civalek and Demir [12] using nonlocal Euler-Bernoulli beam model. 
Janghorban [13] conducted the static and free vibration analysis of carbon nanowires modelled by using Timoshenko beam theory. Differential quadrature method was used in solution process of governing equations. Janghorban and Zare [14] studied the bending of functionally graded single-walled carbon nanotubes (SWCNTs). They used the harmonic differential quadrature method to discretize the governing equations. Akgöz ve Civalek [15] used modified couple stress and strain gradient elasticity theories for bending analysis of SWCNTs. Shaban and Alibeigloo [16] investigated the free vibration and bending behavior of SWCNTs embedded in an elastic medium by using on three-dimensional theory of elasticity. Wu et al. [17] investigated the surface and nonlocal elasticity effects on the elastic behavior of bending nanowires. Akgöz and Civalek [18] studied the static bending response of SWCNTs embedded in an elastic medium based on higher-order shear deformation models and modified strain gradient theory.

In the present paper, static deflection of CNTs under a partial uniform load will be investigated. Effect of nonlocal parameter, nanotube length and uniform load magnitude will be investigated. Results will be depicted in figures. Present results could be useful at design of nano-sensor applications.

\section{Analysis}

A carbon nanotube with length $\mathrm{L}$ and diameter $\mathrm{d}$ is considered. The governing equation of motion for hollow nanotube according to classical continuum mechanics can be obtained as:

$$
E I \frac{\partial^{4} w(x, t)}{\partial x^{4}}=\rho A \frac{\partial^{2} w(x, t)}{\partial t^{2}}+q(x, t)
$$

where $E$ is Young's Modulus, $\rho$ is density, $I$ is moment of inertia, $A$ is cross-sectional area, $w$ is transverse displacement of CNT and $q(x, t)$ is the uniform transverse load.

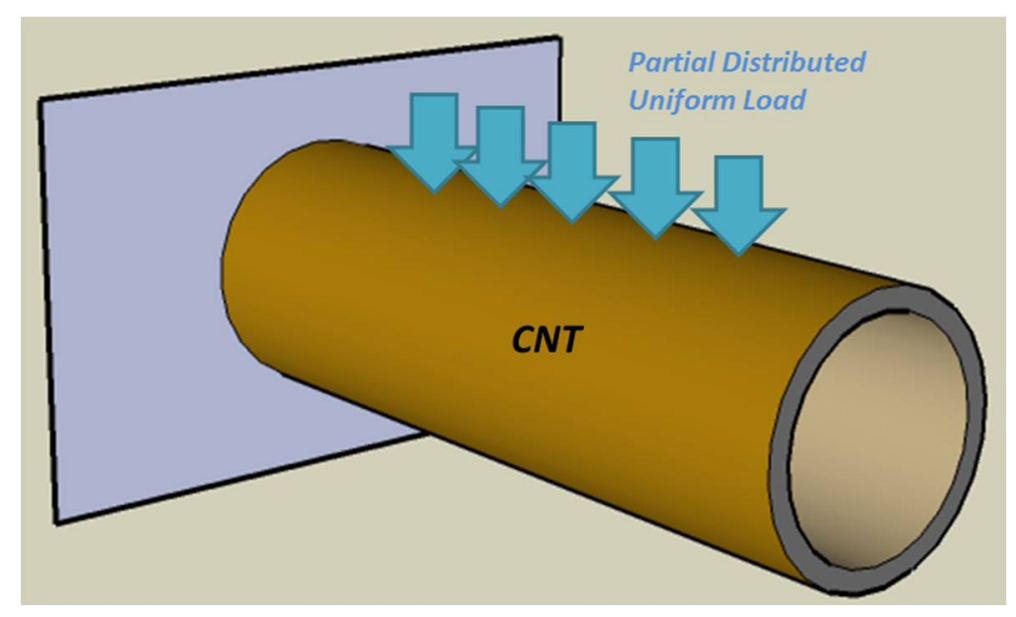

Fig. 1. Continuum Modeling of the Present Problem

\subsection{Governing Equation with Nonlocal Elasticity Theory}

The nonlocal constitute relation can be defined as:

$$
\left(1-\mu \nabla^{2}\right) \tau_{k l}=\lambda \varepsilon_{r r} \delta_{k l}+2 G \varepsilon_{k l}
$$


where $\tau_{k l}$ is the nonlocal stress tensor, $\varepsilon_{k l}$ is the strain tensor, $\lambda$ and $G$ are the Lame constants, $\mu=\left(e_{0} a\right)^{2}$ is called the nonlocal parameter, $a$ is an internal characteristic length and $e_{0}$ is a constant. Eringen determined this parameter as 0.39 for longitudinal wave results according to atomic lattice model [4].

Hooke's Law can be reformulated using the nonlocal elasticity theory in Eq. (2) for uniform $\mathrm{CNT}$ in the one dimensional form:

$$
\left(1-\mu \frac{\partial^{2}}{\partial x^{2}}\right) \sigma=E \varepsilon
$$

where $\varepsilon$ and $\sigma$ are the normal strain and the normal stress of CNT, respectively. If Eq. (3) is inserted into Eq. (1), one obtains [10,15]:

$$
E I \frac{\partial^{4} w(x, t)}{\partial x^{4}}=\left(1-\mu \frac{\partial^{2}}{\partial x^{2}}\right) \rho A \frac{\partial^{2} w(x, t)}{\partial t^{2}}+\left(1-\mu \frac{\partial^{2}}{\partial x^{2}}\right) q(x, t)
$$

Eq. (4) is the governing equation of motion in radial direction for CNT. If the nonlocal parameter is assumed as zero $(\mu=0)$, the classical elasticity equation will be obtained. In static analysis, time derivatives in governing equation are accepted as zero. Eq. (4) is turned into form at below for the static bending analysis of CNT:

$$
E I \frac{\partial^{4} w(x)}{\partial x^{4}}=q(x)-\mu \frac{\partial^{2} q(x)}{\partial x^{2}}
$$

Partial uniform distributed load is considered in the present study. In continuum modeling, partial and point loads can be defined using the Heaviside Step Function and Dirac Delta Function, respectively [19]. If Eq. (5) is rearranged with assumption of dimensionless nanotube length parameter $\left(\bar{x}=\frac{x}{L}\right)$ and partial load case, Eq. (6) is obtained:

$$
\frac{\partial^{4} w(\bar{x})}{\partial \bar{x}^{4}}=\frac{q L^{4}}{E I}\left[H\left(\bar{x}-\alpha_{1}\right)-H\left(\bar{x}-\alpha_{2}\right)\right]-\frac{\mu}{L^{2}} \frac{q L^{4}}{E I} \frac{\partial^{2}}{\partial \bar{x}^{2}}\left[H\left(\bar{x}-\alpha_{1}\right)-H\left(\bar{x}-\alpha_{2}\right)\right]
$$

where dimensionless uniform load can be defined as:

$$
\bar{Q}=\frac{q L^{4}}{E I}
$$

Transverse deflection function of CNT can be found integrating the Eq. (6):

$$
\frac{\partial^{\mathrm{s}} w(\bar{x})}{\partial \bar{x}^{\bar{s}}}=\bar{Q}\left(\bar{x}-\alpha_{1}\right) H\left(\bar{x}-\alpha_{1}\right)-\bar{Q}\left(\bar{x}-\alpha_{2}\right) H\left(\bar{x}-\alpha_{2}\right)-\frac{\mu}{L^{2}} \bar{Q} \frac{\partial H\left(\bar{x}-\alpha_{1}\right)}{\partial \bar{x}}+\frac{\mu}{L^{2}} \bar{Q} \frac{\partial H\left(\bar{x}-\alpha_{2}\right)}{\partial \bar{x}}+C_{1}
$$

$$
\begin{aligned}
& \frac{\partial^{2} w(\bar{x})}{\partial \bar{x}^{2}}=\bar{Q} \frac{\left(\bar{x}-\alpha_{1}\right)^{2}}{2} H\left(\bar{x}-\alpha_{1}\right)-\bar{Q} \frac{\left(\bar{x}-\alpha_{2}\right)^{2}}{2} H\left(\bar{x}-\alpha_{2}\right)-\frac{\mu}{L^{2}} \bar{Q} H\left(\bar{x}-\alpha_{1}\right)+\frac{\mu}{L^{2}} \bar{Q} H\left(\bar{x}-\alpha_{2}\right)+ \\
& C_{1} \bar{x}+C_{2}
\end{aligned}
$$




$$
\begin{aligned}
& \frac{\partial w(\bar{x})}{\partial \bar{x}}=\bar{Q} \frac{\left(\bar{x}-\alpha_{1}\right)^{3}}{6} H\left(\bar{x}-\alpha_{1}\right)-\bar{Q} \frac{\left(\bar{x}-\alpha_{2}\right)^{3}}{6} H\left(\bar{x}-\alpha_{2}\right)-\frac{\mu}{L^{2}} \bar{Q}\left(\bar{x}-\alpha_{1}\right) H\left(\bar{x}-\alpha_{1}\right)+ \\
& \frac{\mu}{L^{2}} \bar{Q}\left(\bar{x}-\alpha_{2}\right) H\left(\bar{x}-\alpha_{2}\right)+C_{1} \frac{\bar{x}^{2}}{2}+C_{2} \bar{x}+C_{3}
\end{aligned}
$$

$$
\begin{aligned}
& w(\bar{x})=\bar{Q} \frac{\left(\bar{x}-\alpha_{1}\right)^{4}}{24} H\left(\bar{x}-\alpha_{1}\right)-\bar{Q} \frac{\left(\bar{x}-\alpha_{2}\right)^{4}}{24} H\left(\bar{x}-\alpha_{2}\right)-\frac{\mu}{L^{2}} \bar{Q} \frac{\left(\bar{x}-\alpha_{1}\right)^{2}}{2} H\left(\bar{x}-\alpha_{1}\right)+ \\
& \frac{\mu}{L^{2}} \bar{Q} \frac{\left(\bar{x}-\alpha_{2}\right)^{2}}{2} H\left(\bar{x}-\alpha_{2}\right)+C_{1} \frac{\bar{x}^{5}}{6}+C_{2} \frac{\bar{x}^{2}}{2}+C_{3} \bar{x}+C_{4}
\end{aligned}
$$

If the Eq. (11) reorganized, Eq. (12) will be obtained:

$$
\begin{aligned}
& w(\bar{x})=\frac{Q}{24}\left[\left(\bar{x}-\alpha_{1}\right)^{4} H\left(\bar{x}-\alpha_{1}\right)-\left(\bar{x}-\alpha_{2}\right)^{4} H\left(\bar{x}-\alpha_{2}\right)\right]-\frac{\mu}{L^{2}} \frac{Q}{2}\left[\left(\bar{x}-\alpha_{1}\right)^{2} H\left(\bar{x}-\alpha_{1}\right)-\right. \\
& \left.q\left(\bar{x}-\alpha_{2}\right)^{2} H\left(\bar{x}-\alpha_{2}\right)\right]+C_{1} \frac{x^{s}}{6}+C_{2} \frac{\bar{x}^{2}}{2}+C_{3} \bar{x}+C_{4}
\end{aligned}
$$

In Eq. (12), $C_{1}, C_{2}, C_{3}$ and $C_{4}$ are the integration constants and can be obtained from boundary conditions. Clamped-Clamped (C-C) and Clamped-Free (C-F) boundary conditions are considered in present study:

$$
\left.\begin{array}{l}
\bar{x}=0 \rightarrow w=\frac{\partial w}{\partial \bar{x}}=0 \\
\bar{x}=1 \rightarrow w=\frac{\partial w}{\partial \bar{x}}=0
\end{array}\right\} \text { Clamped-Clamped }
$$

It is obviously seen that, $C_{3}$ and $C_{4}$ have been found as zero because of clamped boundary condition at $\bar{x}=0 . C_{1}$ and $C_{2}$ have been obtained differently for different boundary conditions as below:

$$
\left.\begin{array}{c}
\frac{C_{1}}{6}+\frac{C_{2}}{2}=\frac{\bar{Q}}{24}\left[\left(1-\alpha_{2}\right)^{4}-\left(1-\alpha_{1}\right)^{4}\right]-\frac{\mu}{L^{2}} \frac{\bar{Q}}{2}\left[\left(1-\alpha_{2}\right)^{2}-\left(1-\alpha_{1}\right)^{2}\right] \\
\frac{C_{1}}{2}+C_{2}=\frac{\bar{Q}}{6}\left[\left(1-\alpha_{2}\right)^{3}-\left(1-\alpha_{1}\right)^{3}\right]-\frac{\mu}{L^{2}} \bar{Q}\left[\left(1-\alpha_{2}\right)-\left(1-\alpha_{1}\right)\right]
\end{array}\right\} C-C
$$



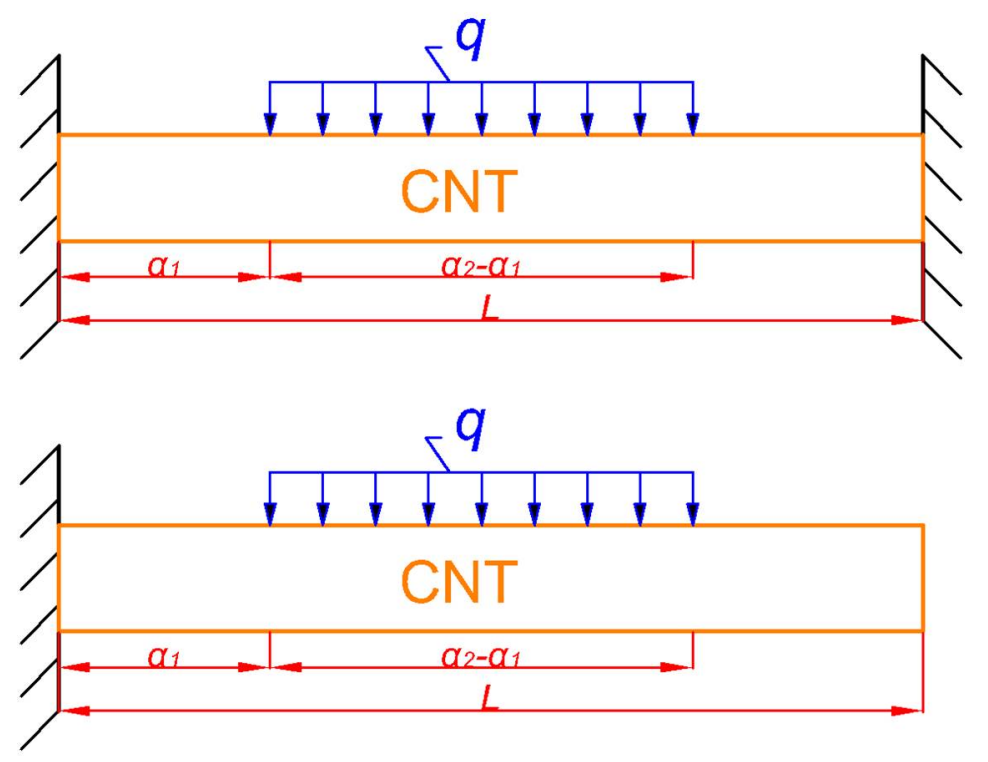

Fig. 2. Partial Uniform Load at C-C and C-F Boundary Conditions

\section{Numerical Results and Discussion}

In this section, nonlocal effect on dimensionless radial deflection of CNT will be investigated. Effects of nonlocal parameter, nanotube length and magnitude of uniform load to dimensionless radial deflection will be depicted in figures.

Numerical results are obtained by independent from material constants. But, interested researchers can use present results in the static bending analysis of CNT which have different chiralities. The assumption in literature for the thickness and elastic properties of the CNT are different. According to Ref. [20,21], Young Modulus $(E)$ and density $(\rho)$ of CNTs do not change substantially for bigger values of diameter of the CNTs $(\mathrm{d} \geq 1 \mathrm{~nm})$.

Nonlocal nanotube model validation can be performed using Lattice Dynamics and MD Simulation results. Eringen [4] used the atomic lattice structure's longitudinal wave results to determine $e_{0}$ and he obtained $e_{0}=0.39$. Nonlocal theory results show good agreement with the Lattice Dynamic.

It can be interpreted that from the Figs. (2-8), nonlocal effect decreases dimensionless radial deflection. However, in longer nanotubes $(\mathrm{L}>20 \mathrm{~nm})$ nonlocal effect vanishes because of the external/internal characteristic length ratio reaches to limit value $(L / a>>1)$. Magnitude of the partial uniform load obviously increases the dimensionless radial deflection and that is an expected result according continuum mechanics.

Position of the uniform distributed load changes the dimensionless radial deflection. The nonlocal theory gives higher deflection values compared with the classical theory for clamped-clamped boundary conditions. It is interesting to note that the nonlocal model gives smaller results compared with classical elasticity theory for cantilever beam bending. 

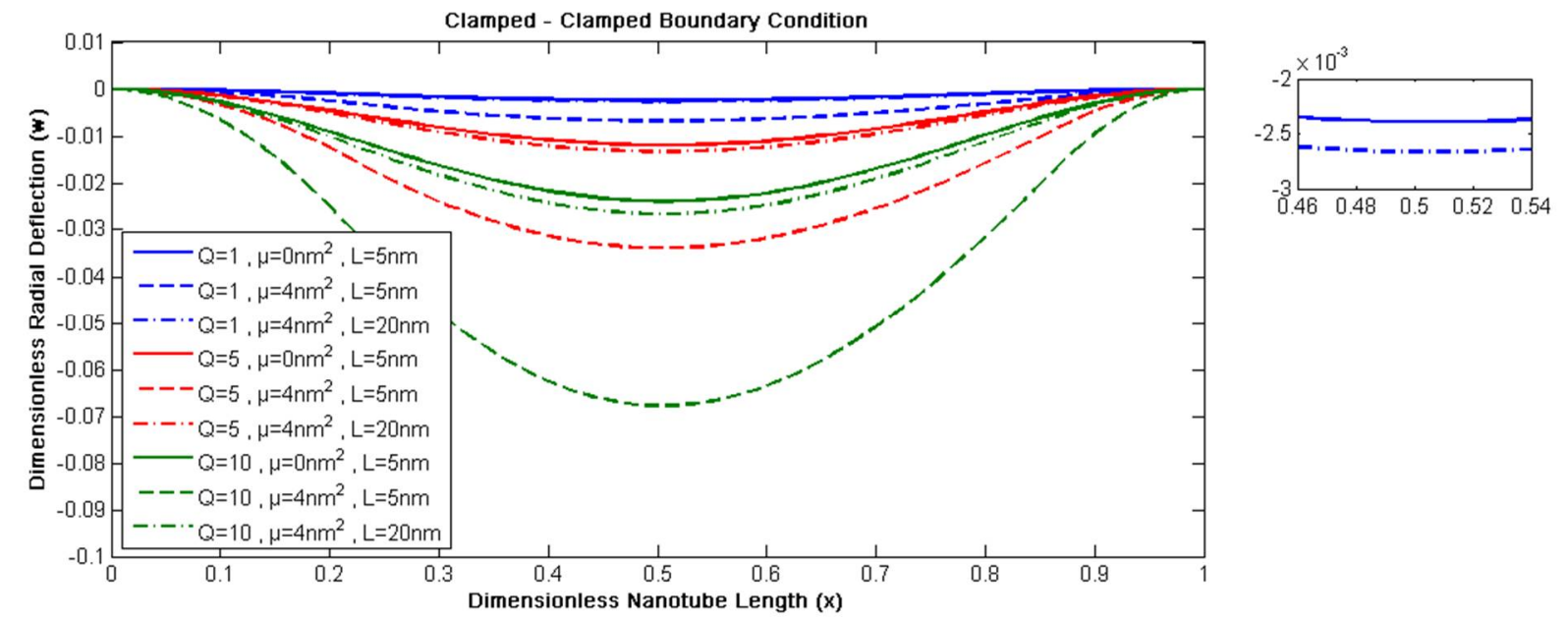

Fig. 3. Changing of Dimensionless Radial Deflection in C-C Case $\left(\alpha_{1}=0.22, \alpha_{2}=0.87\right)$
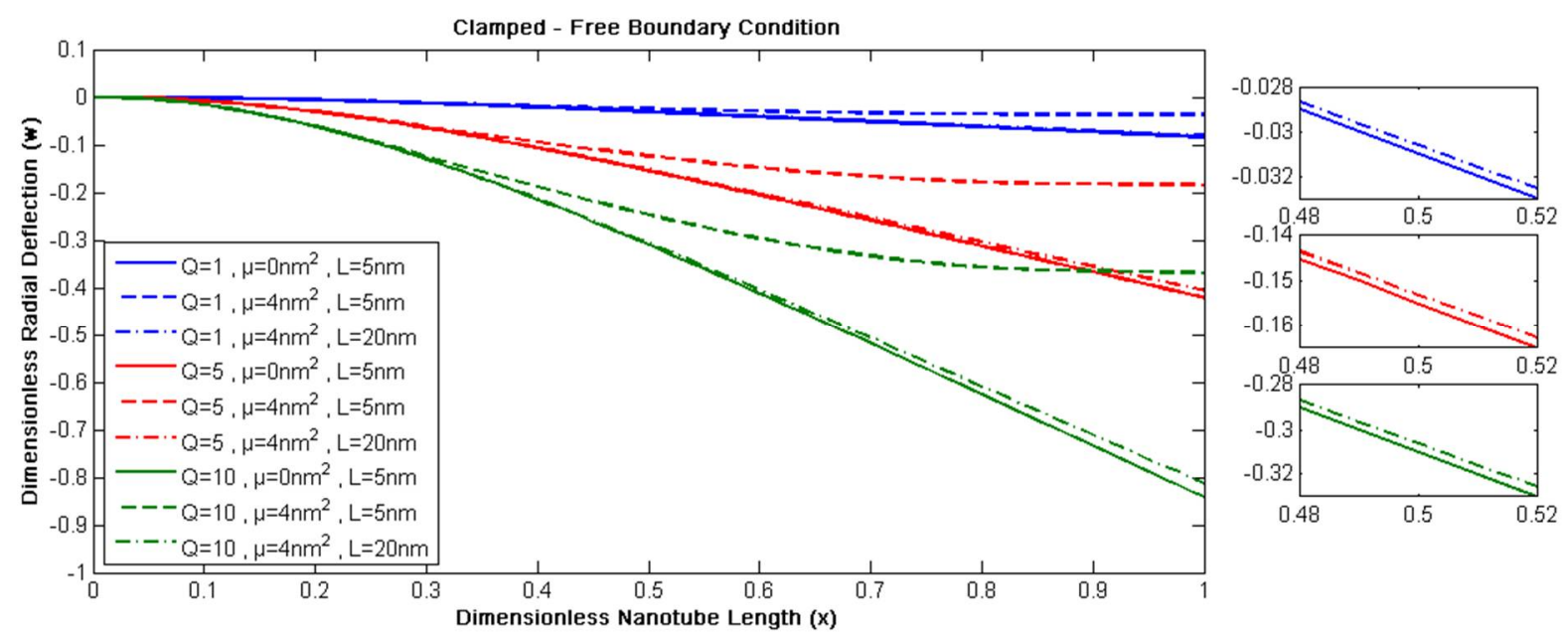

Fig. 4. Changing of Dimensionless Radial Deflection in C-F Case $\left(\alpha_{1}=0.22, \alpha_{2}=0.87\right)$ 

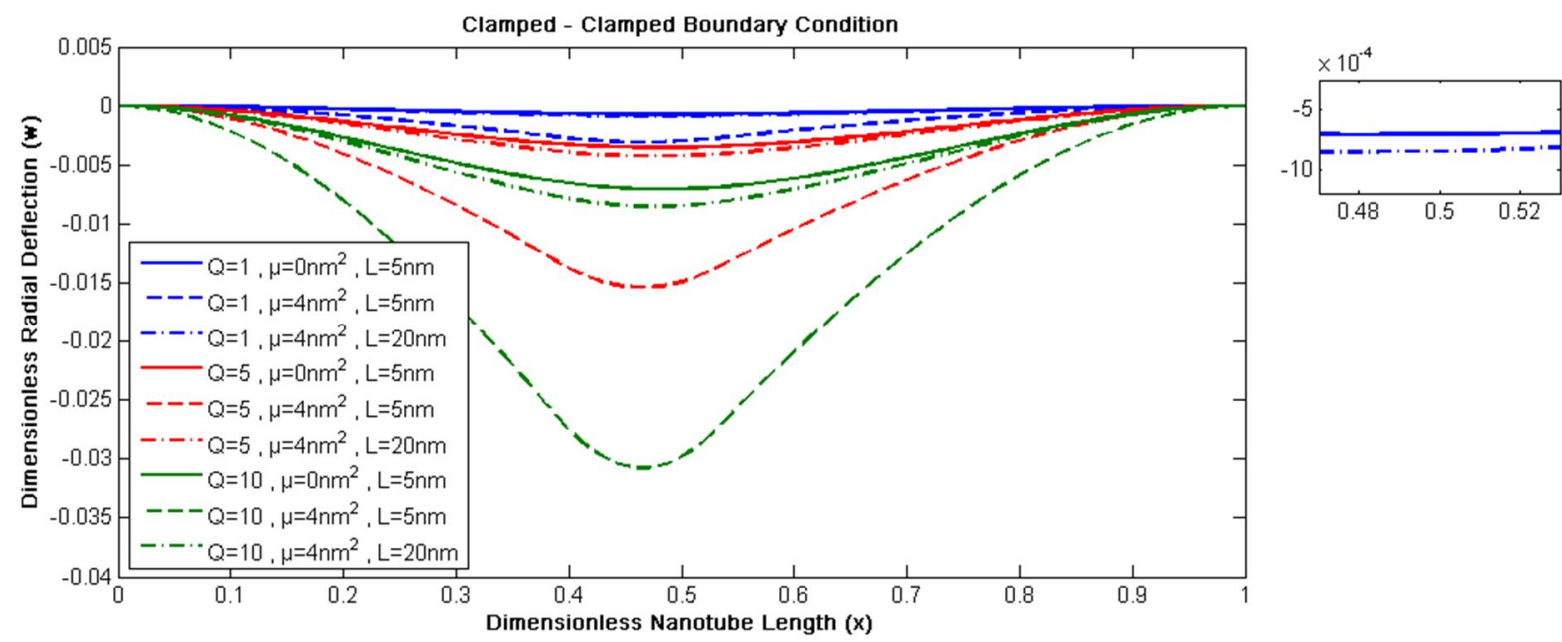

Fig. 5. Changing of Dimensionless Radial Deflection in C-C Case $\left(\alpha_{1}=0.39, \alpha_{2}=0.53\right)$
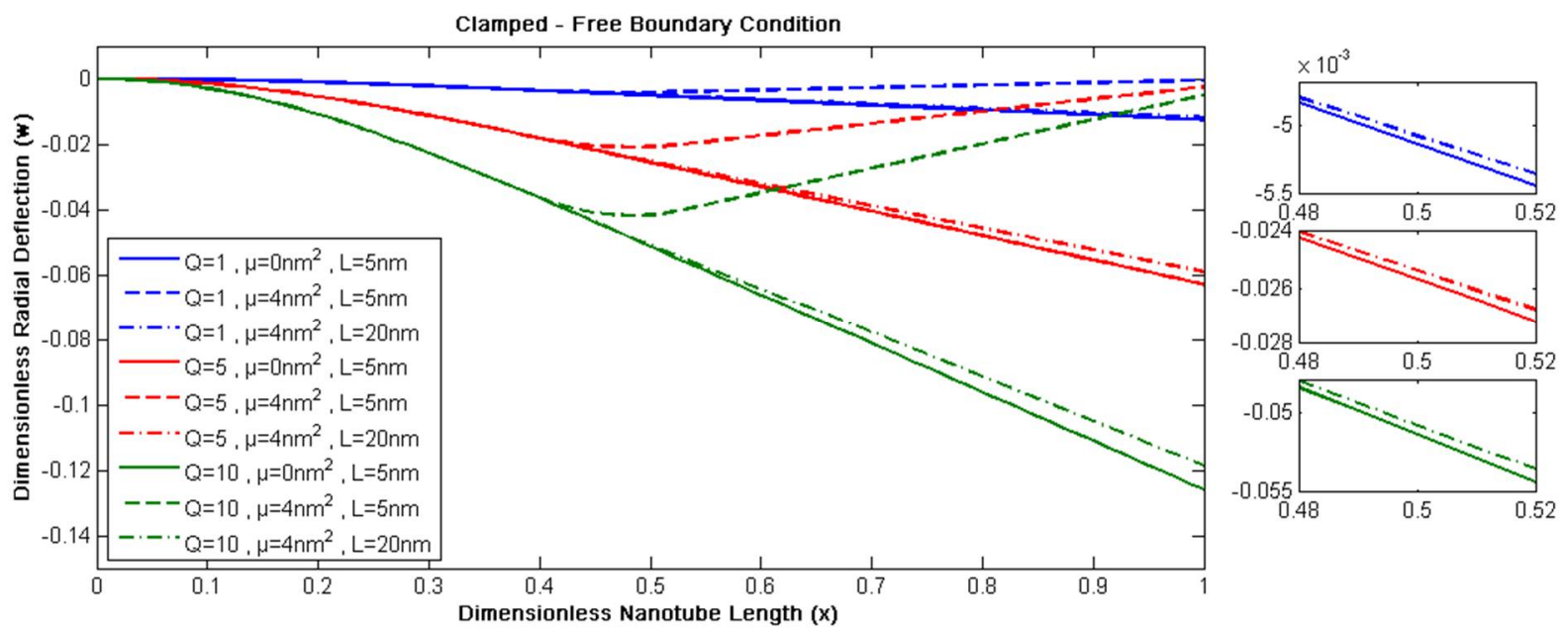

Fig. 6. Changing of Dimensionless Radial Deflection in C-F Case $\left(\alpha_{1}=0.39, \alpha_{2}=0.53\right)$
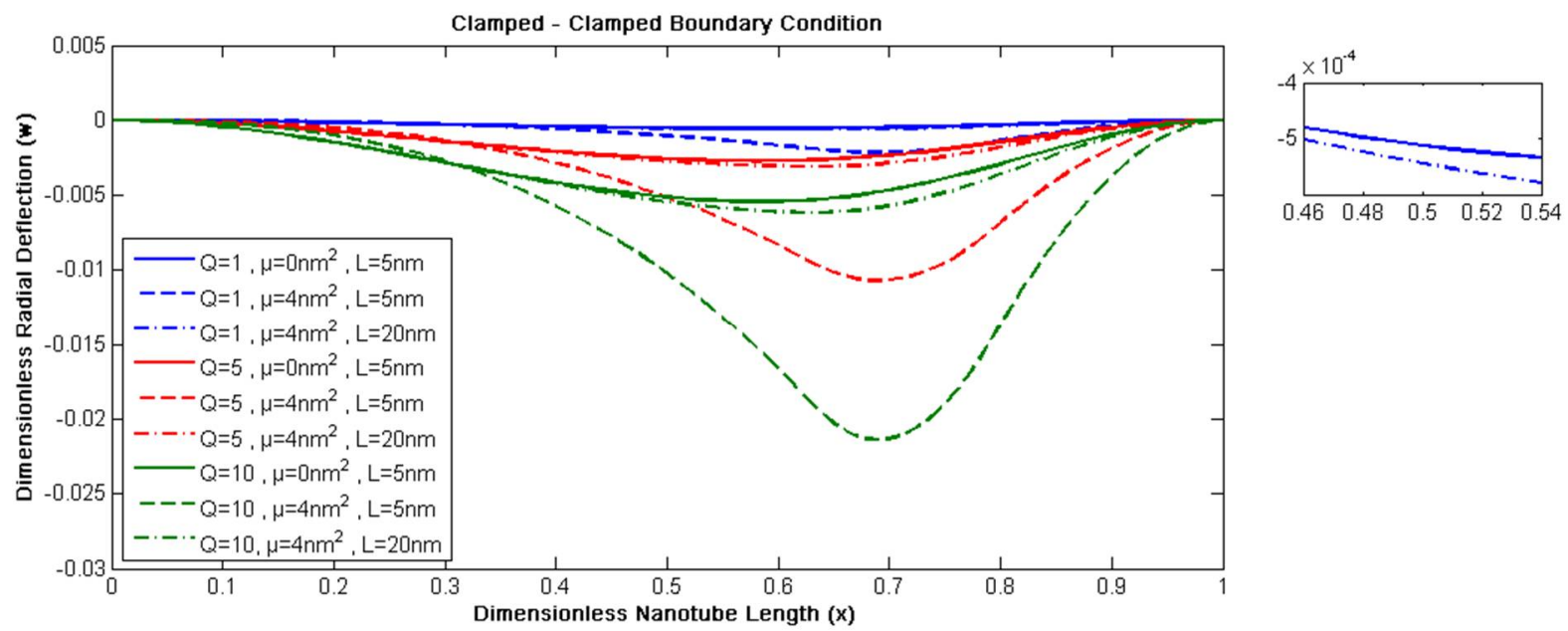

Fig. 7. Changing of Dimensionless Radial Deflection in C-C Case $\left(\alpha_{1}=0.63, \alpha_{2}=0.79\right)$ 


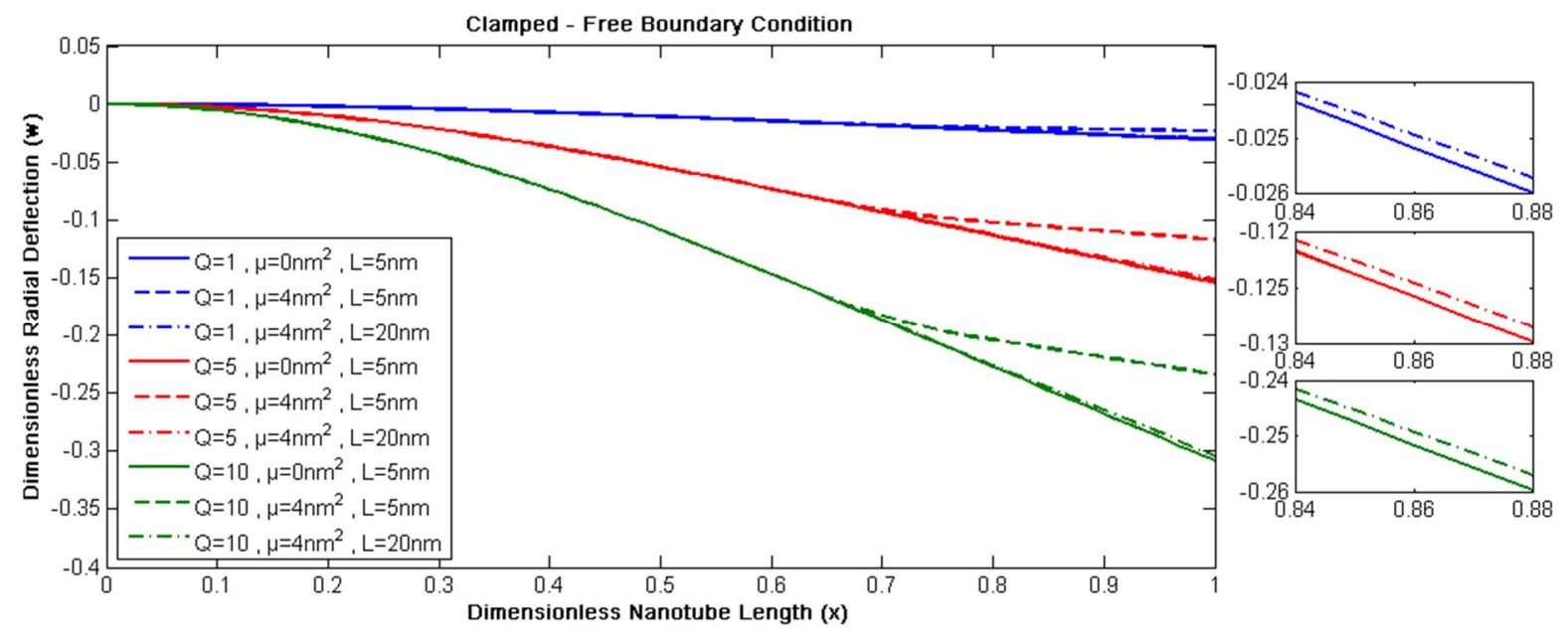

Fig. 8. Changing of Dimensionless Radial Deflection in C-C Case $\left(\alpha_{1}=0.63, \alpha_{2}=0.79\right)$

\section{Conclusion}

In the present work, static radial deflection analysis of CNT under the effect of uniform partial load is investigated. Effects of parameters to the dimensionless radial deflection are obtained. Nonlocal parameter shows decreasing effect on radial deflection and characteristic length ratio determined the effectiveness of nonlocal theory. Magnitude and application area of the load increases the radial deflection of CNT. Present results are non-dimensional and can be used on nano-sensor applications in CNTs which have different chiralities.

\section{REFERENCES}

[1] Elishakoff I. Carbon Nanotubes and Nanosensors: Vibration, Buckling and Balistic Impact. Wiley; 2013

[2] Eringen AC. Linear theory of nonlocal elasticity and dispersion of plane waves, International Journal of Engineering Science, 10, 425-35, 1972. doi:10.1016/00207225(72)90050-X

[3] Eringen AC. Part III - Nonlocal Polar Field Theories. Contin. Phys., 1976, p. 205-67. doi:10.1016/B978-0-12-240804-5.50009-9

[4] Eringen AC. On differential equations of nonlocal elasticity and solutions of screw dislocation and surface waves, Journal of Applied Physics, 54, 4703-10, 1983. doi: $10.1063 / 1.332803$

[5] Dequesnes M, Tang Z, Aluru NR. Static and Dynamic Analysis of Carbon NanotubeBased Switches, Journal of Engineering Materials and Technology, 126, 230, 2004. doi:10.1115/1.1751180

[6] Wang Q, Shindo Y. Nonlocal continuum models for carbon nanotubes subjected to static loading, Journal of Mechanics of Materials and Structures, 1, 663-80, 2006. doi:10.2140/jomms.2006.1.663

[7] Gong N, Liang YC, Yao YX, Liu BG. Static and Dynamic Analysis of Carbon Nanotube Cantilever Based on Molecular Dynamics Simulation, Key Engineering Materials, 375376, 631-5, 2008. doi:10.4028/www.scientific.net/KEM.375-376.631 
[8] Reddy JN, Pang SD. Nonlocal continuum theories of beams for the analysis of carbon nanotubes, Journal of Applied Physics, 103, 2008. doi:10.1063/1.2833431

[9] Aydogdu M. A general nonlocal beam theory: Its application to nanobeam bending, buckling and vibration, Physica E: Low-Dimensional Systems and Nanostructures, 41, 1651-5, 2009. doi:10.1016/j.physe.2009.05.014

[10] Civalek Ö, Demir Ç, Akgöz B. Static analysis of single walled carbon nanotubes (SWCNT) based on Eringen's nonlocal elasticity theory, International Journal of Engineering and Applied Sciences, 47-56, 2009

[11] Arda M, Aydogdu M. Torsional statics and dynamics of nanotubes embedded in an elastic medium, Composite Structures, 114, 80-91, 2014. doi:10.1016/j.compstruct.2014.03.053

[12] Civalek Ö, Demir C. Buckling analysis of cantilever carbon nanotubes using the strain gradient elasticity and modified couple stress theories, Journal of Computational and Theoretical Nanoscience, 8, 1821-7, 2011

[13] Janghorban M. Static and free vibration analysis of carbon nano wires based on Timoshenko beam theory using differential quadrature method, Latin American Journal of Solids and Structures, 8, 463-72, 2011

[14] Janghorban M, Zare A. Harmonic differential quadrature method for static analysis of functionally graded single walled carbon nanotubes based on Euler-Bernoulli beam theory, Latin American Journal of Solids and Structures, 9, 633-41, 2012

[15] Akgöz B, Civalek Ö. Investigation of Size Effects on Static Response of Single-Walled Carbon Nanotubes Based on Strain Gradient Elasticity, International Journal of Computational Methods, 9, 1240032, 2012. doi:10.1142/S0219876212400324

[16] Shaban M, Alibeigloo A. Three dimensional vibration and bending analysis of carbon nano- tubes embedded in elastic medium based on theory of elasticity, Latin American Journal of Solids and Structures, 2122-40, 2014

[17] Wu Q, Volinsky AA, Qiao L, Su Y. Surface effects on static bending of nanowires based on non-local elasticity theory, Progress in Natural Science: Materials International, 25, 520-4, 2015. doi:10.1016/j.pnsc.2015.09.012

[18] Akgöz B, Civalek Ö. Bending analysis of embedded carbon nanotubes resting on an elastic foundation using strain gradient theory, Acta Astronautica, 119, 1-12, 2016. doi:10.1016/j.actaastro.2015.10.021

[19] Demir Ç, Civalek Ö. Nonlocal Deflection of Microtubules Under Point Load, International Journal of Engineering \& Applied Sciences, 7, 33-9, 2016

[20] Li C, Chou T-W. A structural mechanics approach for the analysis of carbon nanotubes, International Journal of Solids and Structures, 40, 2487-99, 2003. doi:10.1016/S00207683(03)00056-8

[21] Wang CY, Zhang LC. A critical assessment of the elastic properties and effective wall thickness of single-walled carbon nanotubes., Nanotechnology, 19, 75705, 2008. doi:10.1088/0957-4484/19/7/075705 